\title{
Diurnal Rhythms of R-R Interval and R-R Interval Variability in the Young Thoroughbred Horse
}

\author{
Yoshiki YAMAYA ${ }^{1}$, Katsuyoshi KUBO ${ }^{2}$, and Akio AMADA ${ }^{3}$ \\ ${ }^{1}$ Laboratory of Veterinary Surgery, College of Agriculture and Veterinary Medicine, Nihon University, \\ 1866 Kameino, Fujisawa-shi, Kanagawa 252, ${ }^{2}$ Equine Research Institute, Japan Racing Association, \\ 5-27-7 Tsurumaki, Setagaya-ku, Tokyo 154, and ${ }^{3}$ Blood Horse Training Center, 1-1-10 Toranomon, \\ Minato-ku, Tokyo 105, Japan
}

\begin{abstract}
Diurnal rhythms of the mean $R-R$ interval and the coefficient of variation $(C V)$ of $R-R$ intervals in eleven young Thoroughbred horses (2-year-old) were examined by periodic analysis to see whether the periodicity of the rhythms change with growing and physical training. On the diurnal rhythm of the mean $R-R$ interval, a single spectrum (14.5 hr-19.3 hr periodicity) was extracted and the power, amplitude and the middle estimating static of rhythm (MESOR) of this periodicity increased with growing and physical training. On the other hand, two periodicities ( $14.5 \mathrm{hr}-15.8 \mathrm{hr}$ and $5.6 \mathrm{hr}-7.5 \mathrm{hr}$ periodicity) were found in the diurnal rhythm of the $R-R$ interval variability but no alteration with growing and physical training was recognized. This suggests that the diurnal rhythm of the mean $R-R$ interval and that of the $R-R$ interval variability are extracted and reflect the different factors affecting autonomic nervous activity. Monitoring the diurnal rhythm of the $R-R$ interval, furthermore, may enable us to understand the progression of growing and physical training effects.
\end{abstract}

Key words: $R$ - $R$ interval variability, young horse

\author{
J. Equine Sci. \\ Vol. 5 No. 3 \\ pp. 83-86, 1994
}

The heart rate in horses is commonly 30-40 beats/min at rest, $90-100$ beats $/ \mathrm{min}$ after the administration of pharmacologic autonomic nervous blockade [9] and over 220 beats/min during submaximum exercise. The change in the heart rate is usually regulated by the autonomic nervous system which branches into the heart and for that reason the heart rate is always fluctuating. The fluctuation in the heart rate has many types of periodicity such as circadian and ultradian rhythms, and indicates the variability of the balance between sympathetic and parasympathetic nervous activity in humans [13, 18]. We have already reported a periodic analysis of the diurnal rhythm of the equine heart rate by means of maximum entropy spectral analysis (MESA) [17]. As MESA is able to detect the characteristics of the fluctuation in the diurnal rhythm of the heart rate, we expected that the frequency and power calcu-

This article was submitted August 23, 1994, and was accepted November 11, 1994.

*corresponding author. lated from the MESA spectrum reflect the autonomic nervous function.

As 2 year-old horses, intensive physical training is begun on the Japanese farm. It is considered that autonomic nervous function changes greatly with physical training and growing. Although Matsui et al. have reported that foals are regulated more sympathetically than mares[8], the process of change in the autonomic nervous function from the young to the adult is not understood.

In this paper, the diurnal rhythm of the R-R intervals in horses was examined by MESA and the change in periodicity accompanying growing and physical training was observed.

\section{Materials and Methods}

Eleven Thoroughbred horses (five males and six females) were examined. Electrocardiograms (ECG) of these horses were recorded intermittently for 30 seconds at 10 minute intervals with a ECG 
recorder (Cardio Tape System, Fukuda Denshi Co., Japan) from 15:00 to 8:00 in January (20 \pm 1 month-old, $441 \pm 24 \mathrm{~kg}$ ), May (24 \pm 1 month-old, $445 \pm 28 \mathrm{~kg})$ and August (27 \pm 1 month-old, $450 \pm$ $33 \mathrm{~kg}$ ) of 1991. The ECG lead was a bipolar chest lead (positive electrode on the left thorax of the highest point of the olecranon and negative electrode on the left side of withers). The horses were individually stabled and could move freely in the standard stall. The feeding times were 6:00 and 16:30, and water was given ad libitum. Training started at 9:00 and ended by 11:00 from Monday to Friday.

After the recording, the tape was played back on an ECG recorder and the R-R interval was measured with an R-R interval analyzer (TM-55, Cerx Co., Japan). The mean R-R interval and the R-R interval variability, which is the coefficient of variation $(\mathrm{CV})$ of the R-R intervals, were calculated from the data for 30 seconds at 10 minute intervals. The time series data for MESA were obtained from means of the mean $\mathrm{R}-\mathrm{R}$ interval and R-R interval variability for eleven horses. Diurnal fluctuations in the mean R-R interval and $\mathrm{R}-\mathrm{R}$ interval variability were analyzed by MESA and the periodicity in those rhythms was extracted. In this study, the values obtained by dividing the number of data by two were used for the order of auto-regression model. The power (spectral density) was normalized by total sample variance. In addition, the amplitude (width between the acrophase and MESOR) and the MESOR (middle estimating static of rhythm) of earned periodicity were calculated by the nonliner cosine fitting method [12].

\section{Results}

The horses had been given the standard exercise test before the ECG recordings. The relationship between running speed $(\mathrm{X}: \mathrm{m} / \mathrm{min})$ and heart rate (Y: beats $/ \mathrm{min})$ was shown as $\mathrm{Y}=0.23 \mathrm{X}+6.10$ ( $\mathrm{X}$ values ranged from 350 to 600 $\mathrm{m} / \mathrm{min}$ ) in January, $\mathrm{Y}=0.24 \mathrm{X}+46.14$ ( $\mathrm{X}$ values ranged from 350 to $600 \mathrm{~m} / \mathrm{min}$ ) in March, $\mathrm{Y}=0.21 \mathrm{X}+53.08$ ( $\mathrm{X}$ values ranged 500 to 800 $\mathrm{m} / \mathrm{min}$ ) in May and $\mathrm{Y}=0.18 \mathrm{X}+68.69$ ( $\mathrm{X}$ values ranged from 500 to $800 \mathrm{~m} / \mathrm{min}$ ) in July. These data indicated that the response of the heart rate to running speed gradually decreased from Janu-

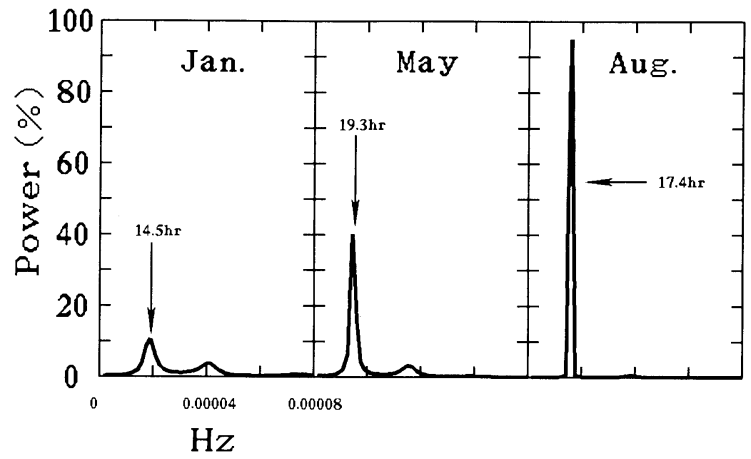

Fig. 1. Power spectrums of the mean R-R interval diurnal rhythm.

By maximum entropy spectral analysis, one spectral peak appeared each month. These pointed out a 14.5 hr-period in Jan., 19.3 hr-period in May and 17.4 hr-period in Aug.

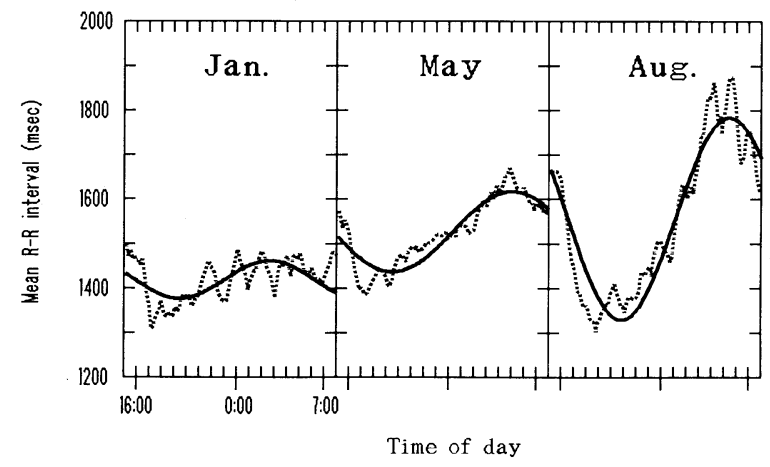

Fig. 2. Diurnal rhythms of mean R-R interval (dot) and cosine fitting curves (solid) (see text for details).

ary to July, due to the training effect.

Diurnal rhythm of the mean $R-R$ interval. According to MESA, the periodicity and power of the spectral peak in the mean $\mathrm{R}-\mathrm{R}$ interval showed a 14.5 hr-period and $10.6 \%$ in January, $19.3 \mathrm{hr}$-period and $40.2 \%$ in May and 17.4 hr-period and $95.1 \%$ in August (Fig. 1).

By the cosine fitting method, the mean R-R interval maximally shortened about 19:00 to 21:00 and lengthened toward about 3:00 to 5:00 in these three months. The amplitude and the MESOR of periodicity were $42.8 \mathrm{msec}$ and $1418.7 \mathrm{msec}$ in January, $89.8 \mathrm{msec}$ and $1526.3 \mathrm{msec}$ in May and $226.9 \mathrm{msec}$ and $1555.5 \mathrm{msec}$ in August (Fig. 2).

Diurnal rhythm of the $R-R$ interval variability. In the $\mathrm{R}-\mathrm{R}$ interval variability, two spectral peaks were recognized by MESA and these periodicities and powers indicated a $15.8 \mathrm{hr}$-period and $9.3 \%$ and 


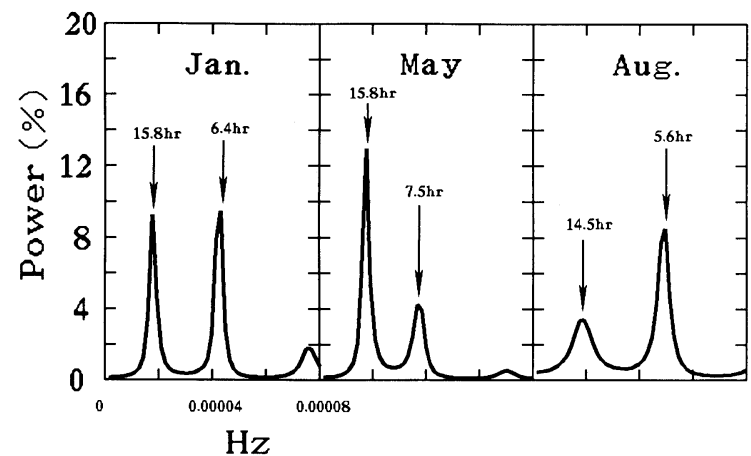

Fig. 3. Power spectrums of the R-R interval variability diurnal rhythm.

By maximum entropy spectral analysis, two spectral peaks appeared each month. These pointed out a 15.6 $\mathrm{hr}$ and $6.4 \mathrm{hr}$-period in Jan., $15.8 \mathrm{hr}$ and $7.5 \mathrm{hr}$-period in May and $14.5 \mathrm{hr}$ and $5.6 \mathrm{hr}$-period in Aug.

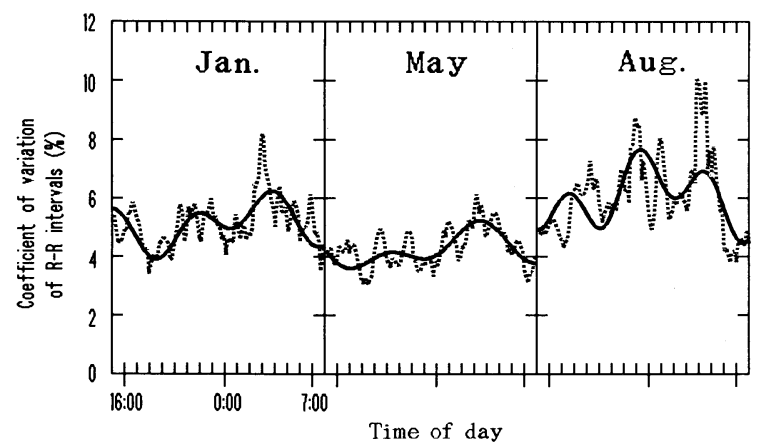

Fig. 4. Diurnal rhythms of $R-R$ interval variability (dot) and cosine fitting curves (solid) (see text for details).

6.4 hr-period and 9.5\% in January, $15.8 \mathrm{hr}$-period and $13.0 \%$ and $7.5 \mathrm{hr}$-period and $4.2 \%$ in May and $14.5 \mathrm{hr}$-period and $3.4 \%$ and $5.6 \mathrm{hr}$-period and 8.5\% in August (Fig. 3).

R-R interval variability decreased about 17:00 to 20:00 and increased toward about 3:00 to 5:00 in these three months. The amplitudes and the MESORs for two periodicities were $0.6 \%$ and $5.1 \%$ (15.8 hr-period) and $0.6 \%$ and $5.1 \%(6.4 \mathrm{hr}-$ period) in January, $0.6 \%$ and $4.3 \%$ (15.8 hrperiod) and $0.4 \%$ and $4.3 \%$ ( $7.5 \mathrm{hr}$-period) in May and $0.8 \%$ and $6.1 \%$ ( $14.5 \mathrm{hr}$-period) and $0.9 \%$ and $6.0 \%$ (5.6 hr-period) in August (Fig. 4).

\section{Discussion}

In man, the assessment of autonomic nervous function by using the minimal hourly heart rate has been reported [11]: The minimal hourly heart rate was lower in young joggers than in young healthy volunteers and also in young healthy volunteers than in old healthy volunteers. This suggests that both sympathetic and vagal tones are decreased by aging and the lower minimal hourly heart rate in young joggers is due to the effect of predominant vagal tone. In this study, the periodicity of the diurnal rhythm of the mean R-R interval is lower than the $24 \mathrm{hr}$-period because of the short recording time (17 hours, 15:00-8:00). The power, amplitude and MESOR of the diurnal rhythm of the mean R-R interval increased with training and growing due to the progressive prolongation of the minimal mean R-R interval in the morning. It may be presumed that the autonomic nervous function regulating the diurnal rhythm of the heart rate is changing widely and trying to adapt to physical training as 2-year-old horses. It has been observed, furthermore, that the resting heart rate of newborn foals is regulated more sympathetically than that of the mares [8]. We think therefore that the increase in parasympathetic tone and/or the decrease in sympathetic tone greatly participated in the formation of the diurnal rhythm of the heart rate.

In healthy horses, bradyarrhythmia has been commonly found on routine clinical examination $[3,6,10]$, but no such arrhythmia was recognized in this study. It is generally known that this phenomenon is induced by increase in vagal tone resulting from training in human subjects $[4,5$, 15]. It has been reported that the bradycardia observed in athletes also occurs due to the decrease in the intrinsic cardiac rate [7]. It therefore suggests that not only the autonomic nervous functions but also the intrinsic heart functions may affect the formation of the diurnal rhythm of the heart rate as 2-year-old horses.

According to MESA, two periodicities were extracted from the diurnal rhythm of the R-R interval variability, whereas only one peak in the spectrum appeared in the diurnal rhythm of the mean R-R interval. The circadian rhythm of heart rate is controlled by the autonomic nervous system through the hypothalamus of the central nervous system in rats [18]. The coefficient of variation (CV) of the R-R intervals also shows a circadian rhythm in man [13] and predominantly reflects parasympathetic activity. Therefore, the CV of the $\mathrm{R}-\mathrm{R}$ intervals is used in assessing autonomic nervous function in diabetic autonomic nervous 
disorders [16]. In addition, it has been believed that beat-to-beat fluctuations in hemodynamic parameters reflect the dynamic response of the cardiovascular control systems to a host of naturally occurring physiological perturbations [1].

Equine studies demonstrated that horses can drowse in the standing position and repeatedly alternate between waking and sleeping during a day [2]. During drowsiness, the gradual lowering of the head progressively reduces the tonic activity of the neck muscle. The R-R intervals in man vary with changes in posture, i.e. the $R-R$ interval variability decreased with postural changes from supine to standing [14]. Therefore, it is expected that the diurnal rhythms of R-R interval variability may be modified by both central and peripheral autonomic nervous activities.

Consequently, the present study suggested that the diurnal rhythms of the mean R-R interval and $\mathrm{R}-\mathrm{R}$ interval variability reflect the different factors which can influence the autonomic nervous activity and the central nervous function in autonomic regulation varies to a great extent through growing and training as 2-year-old horses. Furthermore, monitoring the diurnal rhythm of the R-R interval may enable us to understand the progression of growing and physical training effects.

\section{References}

1. Akselrod, A., Gordon, D., Ubel, F.A., Shannon, D.C., and Cohen, R.J. 1981. Power spectrum analysis of heart rate fluctuation: A quantitative probe of beat-to-beat cardiovascular control. Science 213: 220-222.

2. Dallaire, A. 1986. Rest behavior. Vet. Clin. North Am.: Equine Pract. 2: 591-607.

3. Detweiler, D.K., and Patterson, D.F. 1972. The cardiovascular system. pp. 277-347. In: Equine Medicine and Surgery, 2nd ed. (Catcott, E.J., and Smithcors, J.F. eds.), American Veterinary Publications, Inc., Illinois.

4. Ekblom, B., Kilbom, Å., and Stoltysiak, J. 1973. Physical training, bradycardia and the autonomic nervous system. Scand. J. Clin. Lab. Invest. 32: 251256.

5. Frick, M.H., Elovaino, R.O., and Somer, T. 1967.
The mechanism of bradycardia evoked by physical training. Cardiologia 51: 46-54.

6. Hilwig, R.W. 1983. Cardiac arrhythmias. pp. 131-141. In: Current Therapy in Equine Medicine, 1st ed. (Robinson, N.E. ed.), W.B. Saunders Co., Philadelphia.

7. Katona, P.G., McLean, M., Dighton, D.H., and Guz, A. 1982. Sympathetic and parasympathetic cardiac control in athletes and non athletes at rest. J. Appl. Physiol. 52: 1652-1657.

8. Matsui, K., Sugano, S., and Amada, A. 1986. Heart rate and ECG response to twitching in Thoroughbred foals and mares. Jpn. J. Vet. Sci. 48: 305-312.

9. Matsui, K., and Sugano, S. 1989. Relation of intrinsic heart rate and autonomic nervous tone to resting heart rate in the young and the adult of various domestic animals. Jpn. J. Vet. Sci. 51: 29-34.

10. McGuirk, A.M., and Muir, W.W. 1985. Diagnosis and treatment of cardiac arrhythmias. Vet. Clin. North Am.: Equine Pract. 1: 353-370.

11. Musha, H., Murayama, M., Ito, H., Ono, S., Itai, T., and Kawahara, T. 1986. Estimation of autonomic nervous tone by evaluating minimal hourly heart rate. Kokyu to Junkan 34: 1003-1007 (in Japanese).

12. Otsuka, K., Watanabe, H., and Ogura, H. 1989. Application of the MEM, FFT and Cosinor analyses to chronobiogy in medicine. The Autonomic Nervous System 26: 512-522 (in Japanese).

13. Otsuka, K., Watanabe, H., and Ogura, H. 1990. Circadian variation of heart rate and heart rhythmicity. Kokyu to Junkan 38: 621-628 (in Japanese).

14. Pomeranz, B., Macaulay, R.J.B., Caudill, M.A., Kutz, I., Adam, D., Gordon, D., Kilborn, K.M., Barger, A.C., Shannon, D.C., Cohen, R.J., and Benson, H. 1985. Assessment of autonomic function in human heart rate spectral analysis. Am. J. Physiol. 249: H867-H875.

15. Scheuer, J., and Tipton, C.M. 1977. Cardiovascular adaptations to physical training. Annu. Rev. Physiol. 39: 221-251.

16. Wheeler, T., and Watkins, P.J. 1973. Cardiac denervation in diabetes. Br. Med. J. 4: 584-586.

17. Yamaya, Y., Kubo, K., Amada, A., and Sato, K. 1993. Criterion for maximum entropy spectral analysis of heart rate diurnal rhythm in a horse. Jpn. J. Equine Sci. 4: 73-77.

18. Yanaga, T., and Otsuka, K. 1989. Chronobiological approach to cardiovascular disease. Igaku no Ayumi 148: 713-716 (in Japanese). 